\title{
Moving Dipole Determination From 12-Lead ECGs Can Improve Detection of Acute Myocardial Ischemia
}

\author{
Vito Starc ${ }^{1}$, Todd T Schlegel ${ }^{2}$ \\ ${ }^{1}$ University of Ljubljana, Faculty of Medicine, Ljubljana, Slovenia \\ ${ }^{2}$ Karolinska Institutet, Stockholm, Sweden
}

\begin{abstract}
We investigated the potential clinical utility of a single moving equivalent dipole (MED) constructed from 12lead ECGs. A set of MEDs representing the heart cycle, with each MED comprised of a location, orientation and amplitude, is derived from a spherically bounded volume conductor model using an inverse algorithm. We hypothesized that ischemia could be more sensitively determined by the change in the instantaneous locations of the MED than by changes in the standard scalar ECG.

$M E D$-related data from 103 patients within the STAFF III database who underwent elective coronary artery occlusion (RCA or LCX or LDA) were compared to those from 563 healthy subjects. MED locations and orientations were expressed within the heart's local coordinate system and related to modeled heart wall segments.

Significant changes in MED locations and orientation were noted during coronary artery occlusions, most notably in the LCX and RCA arteries, which are often difficult to detect by standard scalar 12-lead ECG. MED-related changes occurred not only in the ST segment, but also at the end of the QRS. Moreover, MED-related changes occurred very early after occlusion and during low ST segment amplitudes on the scalar ECG, potentially aiding detection of ischemia, especially of the inferior wall.
\end{abstract}

\section{Introduction}

At CinC 2017 we presented an application based on a spherically bounded conductor volume model to determine location, strength, and orientation of a set of single moving equivalent dipoles (MED) representing the heart's electrical activity from 12-lead ECGs [1].

We hypothesized that during myocardial ischemia, with depolarization and/or repolarization changes, it could affect the location of MEDs, revealing the location of ischemia, which might aid its detection. To test our hypothesis we used the STAFF III database with patients who underwent the elective balloon angioplasty with coronary artery occlusion [2].

We sought to determine
1. The behavior of MEDs (their location and orientation) in healthy humans, and

2. Changes in MED trajectory and orientation during occlusion of different coronary arteries, especially in the ST segment and at the end of the QRS.

\subsection{Methods}

\subsection{Determination of a set of MEDs during the heart cycle (the inverse problem)}

To find a set of time-varying MEDs that best describe the measured signals of 12-lead ECG, we applied an inverse algorithm that included the regularization terms to overcome the problem's ill-posedness. As the forward model, we utilized the analytical expression for the potential on a spherical surface generated by a single dipole located within a homogeneous sphere, which enables the optimization of the MED location, without variation of the MED orientation [1]. To reduce possible inaccuracies in the model parameters and measured signals, we included the variation of electrode positions, local conductivities, and slowly varying baseline wander in the optimization algorithm similar to [3]. The error of determination was calculated as the mean difference between the measured and predicted signal.

\subsection{Heart model to assess MED dynamics}

A sphere with radius $\mathrm{R}=15 \mathrm{~cm}$ was fitted to the chest's left anterior surface using anatomical data of an adult torso [4] (Fig. 1). We assumed that each heart follows the mean heart orientation within the thorax, obtained from cardiac MRI studies [4]. The sphere and cardiac MRI data were then used to construct an appropriately oriented biellipsoidal heart with the left (LV) and right (RV) ventricle. The heart's long axis coincided with the LV axis, whereas the short axis perpendicular to the former pointed at the septum center. We also assumed that at the beginning of the QRS, the MED trajectory coincides with the origin of the ellipsoidal LV. 


\subsection{Representation of MEDs in the heart's local coordinate system}

In the short-axis view we determined azimuth $\varphi=0$ in the mid-septum direction and $-180^{\circ} \leq \varphi \leq 180^{\circ}, \varphi$ increasing during clockwise rotation. In the long-axis view we determined elevation $\vartheta=0$ in the equatorial plane and $-90^{\circ} \leq \vartheta \leq 90^{\circ}, \vartheta$ increasing from the basal to the apical orientation. We identified the MED's corresponding segments in a 12-segment LV subdivision (three regions - apical, middle, and basal, and four quadrants - anteroseptal, anterosuperior, lateral and inferior), each with the characteristic coronary artery supply (Fig. 2) [5].

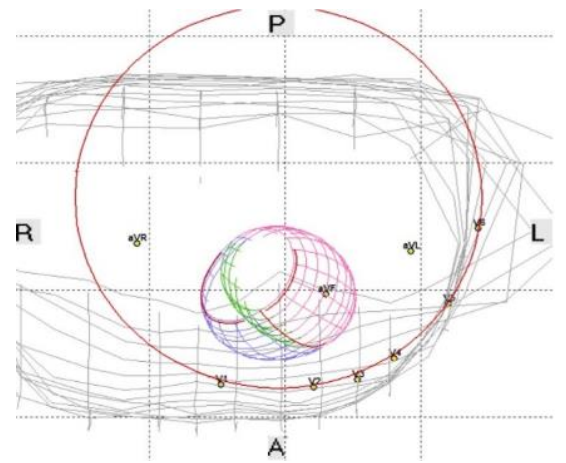

Figure 1 . The positioning of the model heart within the torso

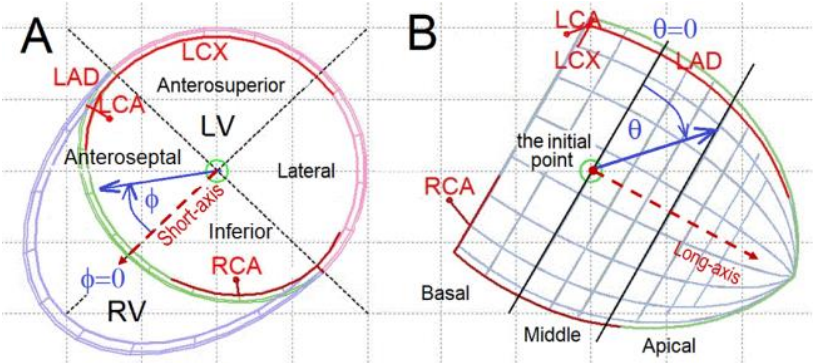

Figure 2. A: The short-axis view the wall segments. B: The long-axis view the regions. RCA, LCA, LAD, and LCX are the coronary arteries.

\subsection{Determination of MEDs from ECG recordings}

All recordings were five-minute (or more) supine resting 12-lead ECGs (sampling rate of $1 \mathrm{kHz}, 300 \mathrm{~Hz}$ low pass filter). From the measurements of the STAFF III study protocol we isolated short segments (20 s) of ECG of the following specimens:

- the baseline, denoted by (0), recorded one day before the catheterization,

- the control (C) before the entrance into the cath-lab,

- before the balloon inflation (B),
- during three instants of inflation (1), (2) and (3), at 1, 3 , and $5 \mathrm{~min}$ of occlusion, respectively, and

- $\quad$ after the balloon inflation (A) and after recovery (R).

We divided the QRS complex of the template into ten segments, QRS0 ... QRS9, each $\sim 10 \mathrm{~ms}$ long. Next, we extracted three ST sections ST1...ST3 at 40, 80, and 120 $\mathrm{ms}$ after the QRS end, respectively, and three $\mathrm{T}$ wave sections, $\mathrm{T} 1 . . \mathrm{T} 3$, before the $\mathrm{T}$ wave peak, at its peak and at the end of the $\mathrm{T}$ wave, respectively, all $20 \mathrm{~ms}$ long.

We used nine signals of the 12-lead ECG (VR, VL, $\mathrm{VF}, \mathrm{V} 1 \ldots \mathrm{V} 6)$ from the above sections to assess MEDs. After identifying beats, we constructed a signal template using signal averaging and delineated the ECG waves. To prevent the $\mathrm{P}$ on $\mathrm{T}$ phenomenon, we recovered the pure $\mathrm{P}$ and $T$ waves by canceling the $T$ wave and the $P$ wave.

\section{Results}

\subsection{The behavior of MEDs in healthy humans}

In our healthy population, the location of MEDs was limited to a small volume, with the orientation nearly constrained within the intraventricular plane as evidenced by two narrow and opposite sectors of MED projections in the short axis view (Fig. 3A). During the QRS-T, the mean direction of the MED described a complete clockwise revolution within the equatorial plane from the initial QRS0 to ST3, pointing at the LAD territory and oriented slightly below the equatorial plane with the final portion of the $\mathrm{T}$ wave pointing at the apex (Fig. 3B).
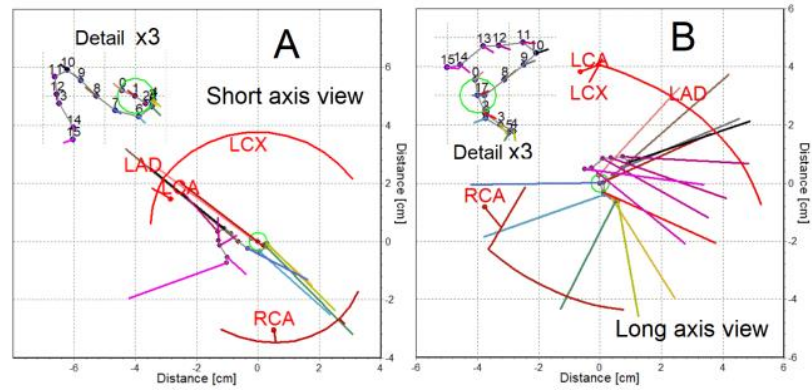

Figure 3. The dynamics of MEDs in a healthy population during the heart cycle. A. The cross-sectional plane. B. The interventricular plane. In both panels at the top/left under the Detail, MED trajectory is enlarged by factor 3. Denotation of the sections: QRS0...QRS9, 0 - 9; ST1...ST3, 10..12; T1..T3, 13...15; Color coding: QRS0...QRS9, from the initial light red changing via yellow, green, and blue to gray; ST1...ST3, black; T1..T3, violet; Each MED represents the mean value of 563 individuals. 

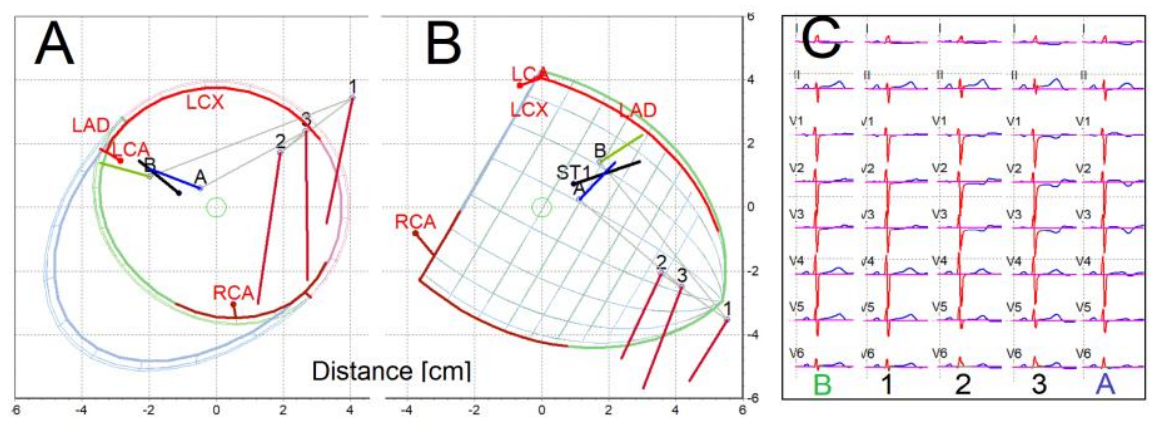

Figure 4. The trend of changes in ST segment MED properties during the proximal occlusion of LCX in different intervention phases. A. The short-axis view; B: The long-axis view; C: 12-lead ECGs of different phases. The phases are denoted as follows: before occlusion "B" in green, during occlusion " 1 ", "2", and "3", for 1, 3, and 5 min of occlusion, respectively, in red, and after occlusion "A" in blue. The MED of ST1 of the control group is in black. On panels A and $\mathrm{B}$, the sequence starts with the green dipole "B", close to and nearly aligned with the healthy group's black dipole. After the first minute of occlusion, it is displaced as red dipole " 1 ", close to and pointing at the LCX territory. It persists there as red dipoles " 2 "and " 3 ", returning close to the initial location and orientation as the blue dipole " $\mathrm{A}$ ".
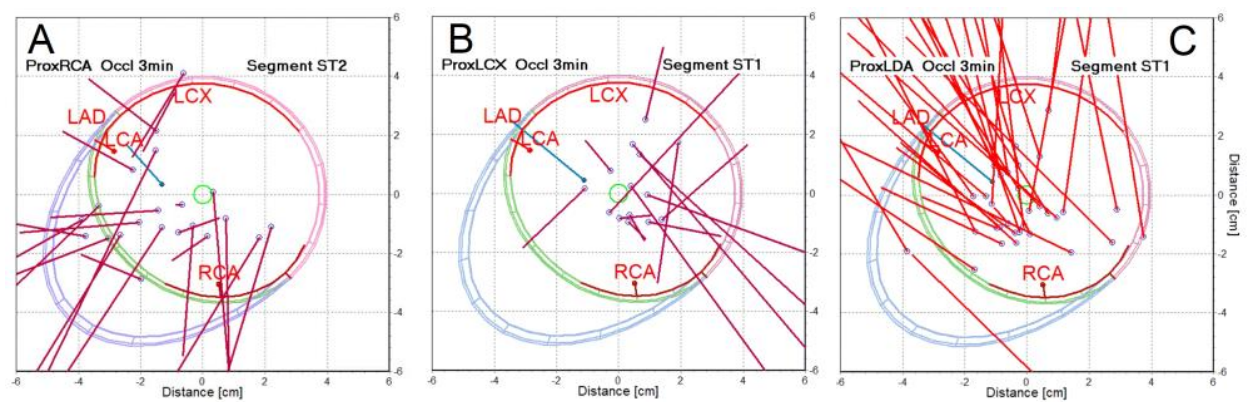

Figure 5. The distribution of MED locations and the ST1 segment's orientation during occlusion of different arteries in the short axis view. A: The occlusion of RCA; B: The occlusion of LCX; C: The occlusion of LAD. During RCA occlusion, the ST1 segment's MEDs were located and oriented predominantly to the inferior and anteroseptal wall; during the LCX occlusion to the lateral wall; and during LAD occlusion anterior to that of our healthy population. The ST1 segment MEDs of the patients are in red, whereas those of controls in blue, pointing to LAD.

\subsection{The behavior of MEDs in healthy humans}

During occlusions of the RCA or LCX arteries, MED properties changed significantly. Besides displaying increased dipolar amplitude, the MEDs of the QRS end and ST segment moved from their initial location (close to the mid-anterior septum) towards the inferior or posterior wall, depending on the culprit artery, orienting themselves to the affected wall segment, then returning close to the previous orientation after recovery. MEDs during the rest (green) versus occlusion (red) conditions were also clearly separable (Fig. 4).In LAD occlusion, the ST segment dipolar amplitude increased, but the change in MED orientation was less notable, presumably due to overlapping with the regular ST segment (Fig. 5 C).

Changes in MEDs were immediate during occlusions, occurring in full extent already at low-grade occlusion in the context of still-unaffected ST segment amplitudes (Figure 4C, note the small ST changes of phase 1 of the occlusion compared to the corresponding notable changes in the MED denoted by "1" in Figures 4A and B).

\subsection{Changes in the MED properties in the late QRS complex}

The observed trajectory displacement of the ST segment during the occlusion towards a new location suggested that the final parts of the QRS may also be affected. We therefore also analyzed MED orientation behavior, specifically changes in the heart's azimuth $\varphi$, during late depolarization and early repolarization, i.e., in the last sections of the QRS and early in the ST segment. The results were expressed as median values for each subgroup. In the control group, $\varphi$ was negative until Q6, i.e., at $\sim 70 \%$ of the ventricular depolarization, pointing to the inferior wall segment. It then reverted to positive values before the QRS end, pointing in the direction of LAD territory (Table I). During RCA and LCX occlusions in STAFF III patients, it remained negative until early repolarization, ultimately pointing toward the LCX rather than LAD territory. Differences between the RCA and LCX subgroups, particularly at the ST segment, followed wall segment-specific orientations noted in Fig. $4 \mathrm{~A}$ and $\mathrm{B}$. 
Table I. The heart's azimuth dynamics towards the QRS end and in the ST segment

\begin{tabular}{lrrrrrrr}
\hline Group & N & Q6 & Q7 & Q8 & Q9 & ST1 & ST2 \\
\hline Control & 511 & $\mathbf{- 6 2}$ & $\mathbf{- 4}$ & 82 & 91 & 98 & 101 \\
prox.RCA & 42 & $\mathbf{- 6 9}$ & $\mathbf{- 5 4}$ & $\mathbf{- 5}$ & 22 & 24 & 52 \\
mid.RCA & 33 & $\mathbf{- 7 6}$ & $\mathbf{- 3 6}$ & $\mathbf{- 1 6}$ & 33 & 42 & 37 \\
dist.RCA & 24 & $\mathbf{- 7 3}$ & $\mathbf{- 6 0}$ & $\mathbf{- 3 4}$ & $\mathbf{- 1 5}$ & $\mathbf{- 8}$ & 46 \\
prox.LCX & 24 & $\mathbf{- 8 4}$ & $\mathbf{- 7 5}$ & $\mathbf{- 5 7}$ & 5 & 12 & 11 \\
mid.LCX & 12 & $\mathbf{- 9 1}$ & $\mathbf{- 6 1}$ & $\mathbf{- 2 7}$ & $\mathbf{- 4}$ & $\mathbf{- 8}$ & $\mathbf{- 1 5}$ \\
dist.LCX & 16 & $\mathbf{- 8 0}$ & $\mathbf{- 6 9}$ & $\mathbf{- 5 9}$ & $\mathbf{- 4 2}$ & $\mathbf{- 4 0}$ & $\mathbf{- 3 4}$ \\
prox.LDA & 56 & $\mathbf{- 6 7}$ & 97 & 101 & 96 & 96 & 98 \\
mid.LDA & 25 & $\mathbf{- 5 9}$ & -26 & 85 & 89 & 91 & 98 \\
\hline
\end{tabular}

Azimuth is in degrees. $\mathrm{N}$, number of participants; Q6...Q9, sections QRS6...QRS9; ST1 and ST2, the first two sections of ST segment; prox., proximal; mid., middle; dist., distal; RCA, LCX and LDA, the coronary arteries; Negative numbers are bold;

\subsection{The patterns of response}

We observed different patterns regarding the extent of change in the MED location and orientation of the ST1 section. To evaluate the former's distribution and differentiate between weak and robust responders, we determined optimal threshold of deviation angle $\alpha$ and distance $\mathrm{dr}$ to best separate the occlusion subgroups from controls. The corresponding threshold values, $\alpha_{\text {Thr }}=60^{\circ}$ and $\mathrm{r}_{\mathrm{Thr}}=3 \mathrm{~cm}$, served to detect responders regarding MED property changes. We found that the most accurate performer among the MED properties was a deviation angle $\alpha$ above the threshold, which occurred in $~ 83-84 \%$ of cases of RCA or LCX occlusions compared to $17 \%$ of controls at baseline (Table II). Much less influential was the distance dr that was also high in the control group, decreasing detection specificity.

Table II. The percentage of each group affected at any time by orientation and location of MEDs above optimal thresholds

\begin{tabular}{lrrrrrr}
\hline & $\mathrm{N}$ & $\% \alpha$ & $\% \mathrm{dr}$ & $\% \alpha \wedge \mathrm{dr}$ & $\% \mathrm{D}$ & $\% \mathrm{ST}$ \\
\hline Control & 563 & $\mathbf{1 7}$ & $\mathbf{4 9}$ & $\mathbf{1 3}$ & $\mathbf{9}$ & $\mathbf{7 6}$ \\
RCA & 145 & 83 & 60 & 56 & 23 & 79 \\
LCX & 92 & 84 & 62 & 45 & 32 & 71 \\
LAD & 137 & 68 & 63 & 52 & 52 & 99 \\
\hline
\end{tabular}

$\mathrm{N}$, number of participants; the percentage of responders for $\alpha>60^{\circ}, \mathrm{dr}>3 \mathrm{~cm}, \mathrm{D}>100$ units, and $\mathrm{ST}>100$ $\mu \mathrm{V} ; \alpha$, the angle between two MED orientations of the occlusion and control; dr, the distance between the two corresponding locations; $\alpha \wedge d r$, conjunction; D, dipole strength in units; ST, non-specific ST segment amplitude.

\section{Discussion and Conclusion}

We describe a new method for detecting early myocardial ischemic changes due to coronary artery occlusion. Based on solving the inverse problem, the method utilizes a spherical bonded model with a homogenous conductor and enables the determination of the moving dipole properties in the heart cycle from the 12-lead ECG. We applied the method to analyze ECGs in the group of patients who underwent elective coronary artery occlusion and in a large control group, with both reaching an error of determination slightly below $5 \mu \mathrm{V}$.

In healthy individuals, we showed that the MED activity is nearly constrained within the intraventricular plane, with MED orientation also rotating clockwise within this plane.

The study showed significant changes in MED location, orientation and amplitude during occlusion of the coronary arteries compared to both a control group and to the control state. The most significant changes in MED occurred during LCX and RCA occlusions, which are sometimes difficult to detect with standard scalar ECG. Changes appeared very early after occlusion, occurring at corresponding low ST segment amplitudes, and were not limited to the ST segment only, also including the last part of the QRS. Despite some variability in the pattern of change in the MED, which require further explication, the method may eventually aid the detection of the inferior wall myocardial ischemia caused by LCX and RCA occlusions.

\section{References}

References:

[1] V. Starc, and C.A. Swenne, "Spatial distribution and orientation of a single moving dipole computed in 12-lead ECGs in a healthy population using a spherically bounded model," Computing Cardiol., vol. 44, pp.1-4, 2017.

[2] S.G. Warren, and G.S. Wagner GS, "The STAFF studies of the first 5 minutes of percutaneous coronary angioplasty balloon occlusion in man", J Electrocardiol, vol. 47, pp.402-407, 2014.

[3] W. Bystricky, "Identification of strict left bundle branch block using a moving dipole model," Computing in Cardiol., vol. 45, pp.1-4, 2018.

[4] F. Odille, S. Liu, P. van Dam, and J. Felblinger, "Statistical. variations of heart orientation in healthy adults," Computing Cardiol., vol. 44, pp.1-4, 2017.

[5] R.H Selvester et al, "Myocardial Infarction", In: P.W. Macfarlane et al. (eds), Comprehensive Electrocardiology. Springer, London 2010.

Address for correspondence:

Vito Starc, MD, $\mathrm{PhD}$

Ljubljana University, Faculty of Medicine

Zaloska 4, SI 1000 Ljubljana, Slovenia

E-mail: vito.starc@mf.uni-lj.si. 